\title{
Bloc biopsy by tunneling method using endoscopic submucosal dissection for an upper gastrointestinal submucosal tumor
}

Minimally invasive local resection techniques such as hybrid natural orifice transluminal endoscopic surgery (NOTES) are becoming established in the treatment of upper gastrointestinal submucosal tumor (UGISMT), especially gastrointestinal stromal tumor (GIST) [1]. However, the tissue obtained from UGISMTs by techniques such as endoscopic ultrasound (EUS)-guided biopsy is occasionally insufficient to make a diagnosis [2]. Therefore, we devised a safe and reliable technique for bloc biopsy by a tunneling method [3, 4] using endoscopic submucosal dissection (ESD) [5]. Here, we describe the methodology of this technique.

A 73-year-old man was found on endoscopy to have a 25-mm gastric submucosal tumor ( Fig. 1 a), which was shown on EUS to be originating from the muscularis propria. The following procedure was performed after the patient had given informed consent.

After marking the mucosa around the tumor with a margin of about $10 \mathrm{~mm}$, a small incision was made to create a $10-\mathrm{mm}$ opening, and the submucosa was approached through the opening (the ESD procedure). The short tunneling method

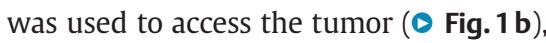
which was visually identified and decapsulated ( $\bullet$ Fig.1 c). A needle-knife for ESD in cutting mode was used to obtain a $5 \times 5 \times 2-\mathrm{mm}$ bloc biopsy specimen ( $\bullet$ Fig.1d). The tissue was collected into a long attachment (Elastic Touch F-01; Top Corporation, Tokyo, Japan) with care being taken that the tissue did not come into contact with the inner wall of the tunnel. The entire exposed surface was sutured with clips that were placed starting at the far end of the tunnel and finishing at the opening to prevent any tumor fragments flowing back into the tunnel ( $\bullet$ Fig. 1 e, $\diamond$ Video 1 ).

\section{Video 1}

Endoscopic views of a bloc biopsy being performed by the tunneling method using endoscopic submucosal dissection (ESD) for an upper gastrointestinal submucosal tumor.
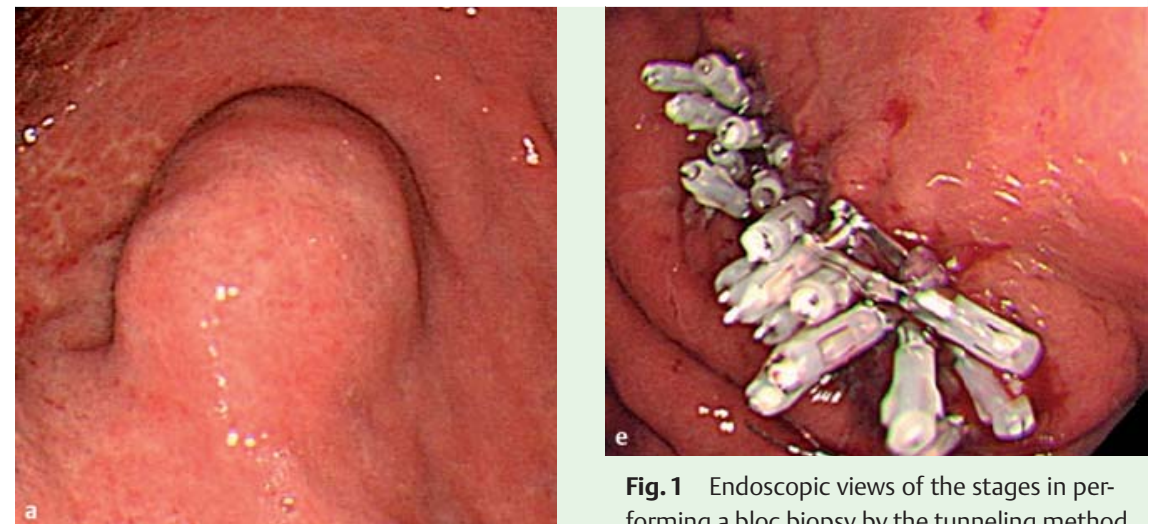

Fig. 1 Endoscopic views of the stages in performing a bloc biopsy by the tunneling method using endoscopic submucosal dissection for an upper gastrointestinal submucosal tumor showing: a a $25-\mathrm{mm}$ gastric submucosal tumor on the posterior wall of the upper gastric corpus, which was protruding into the lumen; b the 10-mm opening through which a tunnel was made to separate the submucosa from the tumor; c a whitish tumor that was protruding into the submucosa, which was identified visually and decapsulated (red arrows); d a needle-knife in cutting mode being used to separate a bloc specimen $(5 \times 5 \times 2 \mathrm{~mm}$; red arrows) under direct vision while minimizing tissue crushing and the long attachment (blue arrows) into which the tissue was then collected using grasping forceps; $\mathbf{e}$ the incision after it had been closed with clips placed from the far end of the tunnel to the opening.

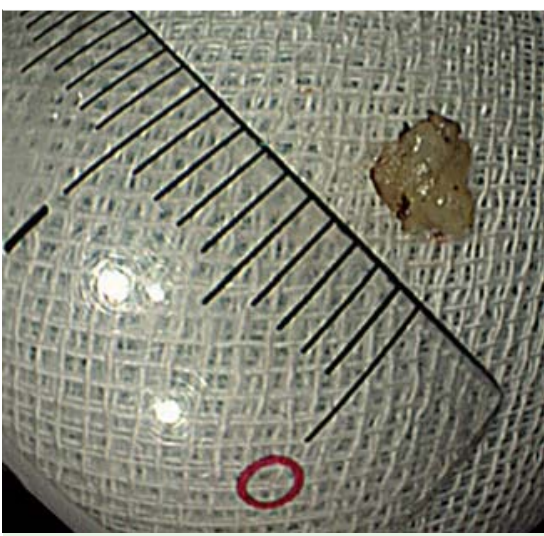

Fig. 2 Macroscopic appearance of the 5-mm tumor specimen obtained, which was of sufficient size to make an immunopathologic diagnosis of gastrointestinal stromal tumor (GIST). 
The entire procedure was completed without complications in 40 minutes. The specimen obtained was sufficient in size ( $5 \mathrm{~mm}$; Fig. 2), and immunopathology was used to make a diagnosis of GIST. This technique enabled us to obtain a histopathologic diagnosis without any complications, such as hemorrhage or dissemination of the tumor.

\section{Endoscopy_UCTN_Code_TTT_1AO_2AC}

Competing interests: None

\section{H. Kobara, H. Mori, S. Fujiwara, N. Nishiyama, M. Kobayashi, T. Masaki}

Department of Gastroenterology and Neurology, Kagawa Medical University School of Medicine, Kagawa, Japan

\section{References}

1 Mori H, Kobara H, Masaki T et al. Establishment of pure NOTES procedure using a conventional flexible endoscope: review of six cases of gastric gastrointestinal stromal tumor. Endoscopy 2011; 43: 1-4

2 Lee CK, Chung IK, Cho HD et al. Endoscopic partial resection with the unroofing technique for reliable tissue diagnosis of upper GI subepithelial tumors originating from the muscularis propria on EUS (with video). Gastrointest Endosc 2010; 71: 188-194

3 Sumiyama K, Gostout CJ, Rajan E et al. Submucosal endoscopy with mucosal flap safety valve. Gastrointest Endosc 2007; 65: 688-694

4 Yoshizumi F, Yasuda K, Kawaguchi $K$ et al. Submucosal tunneling using endoscopic submucosal dissection for peritoneal access and closure in NOTES: a porcine survival study. Endoscopy 2009; 41: 707-711

5 Lee IL, Lin PY, Tung SY et al. Endoscopic submucosal dissection for the treatment of intraluminal gastric subepithelial tumors originating from the muscularis propria layer. Endoscopy 2006; 38: 1024-1028
Bibliography

Dol http://dx.doi.org/

10.1055/s-0031-1291821

Endoscopy 2012; 44: E197-E198

(c) Georg Thieme Verlag KG

Stuttgart · New York

ISSN 0013-726X

\section{Corresponding author}

\section{H. Kobara, MD, PhD}

Department of Gastroenterology and Neurology Kagawa Medical University School of Medicine

1750-1 Ikenobe, Miki, Kita

Kagawa 761-0793

Japan

Fax: +81-87-8912158

kobara@med.kagawa-u.ac.jp 